\title{
Application of microwave motion sensors in navigation systems for ground vehicle
}

\author{
Dmitrii Khablov* \\ V. A. Trapeznikov Institute of Control Sciences IPS RAN, 65 Profsoyuznaya Street, 117997, \\ Moscow, Russia
}

\begin{abstract}
Effective land transport management in a controlled and unmanned mode is impossible without its accurate and continuous positioning. The paper discusses the possibility of increasing this accuracy in the absence or uncertain reception of signals from satellites of the global navigation system. Moreover, the use of an additional self-navigation inertial system to solve this problem in this case is not justified for reasons of accuracy and cost. Therefore, as an alternative autonomous navigation system, a solution based on radar Doppler sensors of modular type is proposed. The methods of measuring the velocity vector and the algorithm of direct continuous measurement of displacements are considered. It is shown that the latter measurement option can significantly reduce the cumulative error when positioning vehicles.
\end{abstract}

\section{Introduction}

The operation of automated control systems in land transport requires reliable, accurate and continuous positioning throughout the designated coverage area. Unfortunately, the global navigation satellite system (GNSS or GLONASS) cannot fully provide these criteria, especially when working indoors and in conditions of poor reception of signals from satellites. This leads to the need to use additional local or autonomous navigation systems, the data from which are used in conjunction with GNSS in the so-called hybrid navigation systems [1]. Normally, an inertial navigation system (INS) is used for this purpose, but it is not effective enough in the case of ground positioning. Its accuracy depends on the mass of the object, friction, while the errors in determining the coordinates are cumulative. INS determines the movement after double integration of accelerometer readings over time and integration of rotational speed sensors along three axes, as a result of which an error accumulates in the intervals between corrections. If this function is not essential for use on an aircraft due to the presence of corrective signals from GNSS satellites, then on land use this can lead to significant errors in positioning. The paper investigates perspective terrestrial positioning system of independent type radar in the millimeter microwaves. It is built by analogy with the Doppler speed and drift angle sensor used in aviation [2]. Microwave sensor signal processing algorithms for obtaining accurate and continuous navigation data are presented.

\footnotetext{
*Corresponding author: dkhablov@mail.ru
} 


\section{Using Doppler microwave sensors in navigation}

\subsection{Inertial navigation system for terrestrial applications}

The inertial navigational system, as noted above, determines the displacement through the double integration of acceleration, therefore, in addition to the effect of the accumulation of error, the accuracy will depend on many parameters. In addition, specific applications in land transport, in contrast to the air adds, new factors affecting the accuracy. Among them are friction forces, adhesion to the surface, air resistance, effective area and weight of the object, a centre of gravity, shaking, a wide range of changes in speed and acceleration. This makes it difficult to use INS in cars as a standalone addition to the global navigation system. At the same time, the radar system used in aviation for measuring the velocity vector and drift angle, which allows adjusting INS data under certain conditions, cannot be used unchanged in the ground version due to the absence of one of the degrees of freedom in space.

\subsection{Ground radar navigation system}

The radar navigation system for ground-based applications is based on the use of the Doppler Effect [2]. Usually, in the simplest case, continuous microwave radiation is used. Two antennas from one point in front or on both sides of the vehicle emit microwaves with a frequency $f_{0}$ forward and at angles $\alpha$ to the direction of motion in the vertical plane and at angles $\beta$ on different sides of its axis in the horizontal plane. Electromagnetic waves reflected from the road surface are received by the same antennas. Then they are mixed with part of the emitted waves, as a result of which two Doppler signals with frequencies fD1 and fD2 are distinguished for the left and right sides. These frequencies are proportional to the velocities $V_{1}$ and $V_{2}$ of the mutual movement of $S_{1}$ and $S_{2}$ between the antennas and the centres of the tracks from the antenna patterns on the surface (Fig. 1a, b):

a)
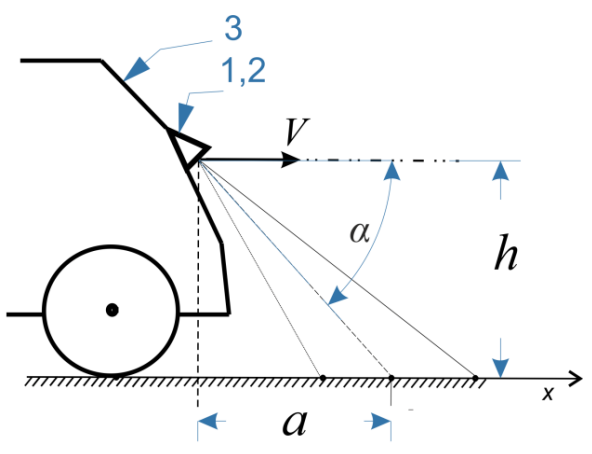

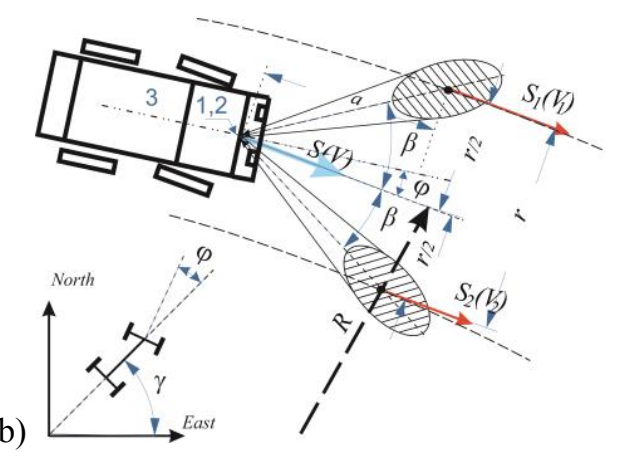

Fig. 1. The location of the antennas of Doppler sensors 1 and 2 on ground transport -3: a) - side view; b) - top view

$$
f_{D 1}=2 V_{1} \cos (\alpha) \cos (\beta) / \lambda_{0} \text { and } f_{D 2}=2 V_{2} \cos (\alpha) \cos (\beta) / \lambda_{0},
$$

where $\lambda_{0}=c / f_{0}$ is the wavelength of electromagnetic waves, and $c$ is the speed of light. Hence, from formulas (1), respectively, you can determine both speeds:

$$
V_{1}=\lambda_{0} f_{D 1} / 2 \cos (\alpha) \cos (\beta) \quad \text { and } \quad V_{2}=\lambda_{0} f_{D 2} / 2 \cos (\alpha) \cos (\beta)
$$


Figure $1 \mathrm{~b}$ shows the phase $\varphi$ of vehicle rotation for a small period of time $\Delta t$ relative to the previous direction (angle $\gamma$ ). Both centres of the tracks from the antenna patterns at this moment in time move along arcs with radii $R+r$ and $R-r$ if the distance between them is $r$, and the centre of the vehicle moves along the radius $R$. As a result of the transformations from (1) and (2), we can obtain the expression for the vehicle velocity vector for each discrete time interval $\Delta t$ in the form of module $V$ and angle $\varphi[3]$ :

$$
\begin{aligned}
& V=\lambda_{0}\left(f_{D 1}+f_{D 2}\right) / 4 \cos (\alpha) \cos (\beta), \\
& \varphi=\lambda_{0}\left(f_{D 1}-f_{D 2}\right) / 4 \pi r \cos (\alpha) \cos (\beta)
\end{aligned}
$$

where $V=\left(V_{1}+V_{2}\right) / 2$. The entire route can be divided into discrete segments $\Delta t_{i}$, where $i=$ $0,1,2 \ldots N$, where $N$ is the total number of such segments. To describe the nature of the movement in this case, you can use a two-dimensional array of these values $\left\{V_{i}, \varphi_{i}\right\}$. The movement at each time interval $\Delta t_{i}$ occurs on the circumference with radius $R_{i}$, and the vehicle moves from point $i$ to $i+1$ chorda $L_{i}$, which are determined from formulas (3):

$$
R_{i}=r\left(f_{D 1 i}+f_{D 2 i}\right) / 2\left(f_{D 1 i}-f_{D 2 i}\right), \quad L_{i}=2 R_{i} \sin \left(\varphi_{i}\right), \quad r=2 h \operatorname{ctg}(\alpha) \operatorname{tg}(\beta) .
$$

As a result, the formulas (3) and (4) give expressions for the route in the North - East coordinates:

$$
\begin{aligned}
& x_{n i}=L_{1} \sin \varphi_{1}+L_{2} \sin \left(2 \varphi_{1}+\varphi_{2}\right)+\ldots+L_{i-1} \sin \left(2 \varphi_{1}+2 \varphi_{2}+\ldots+\varphi_{i-1}\right) \\
& x_{e i}=L_{1} \cos \varphi_{1}+L_{2} \cos \left(2 \varphi_{1}+\varphi_{2}\right)+\ldots+L_{i-1} \cos \left(2 \varphi_{1}+2 \varphi_{2}+\ldots+\varphi_{i-1}\right)
\end{aligned}
$$

\section{Algorithms for calculating instantaneous velocity and movement by Doppler microwave sensors}

\subsection{Instantaneous Doppler frequency and speed measurement}

To determine the route $\left[x_{n}, x_{e}\right]$ it is necessary to accurately measure the values of the instantaneous Doppler frequencies, and to integrate them in time. In [3], an algorithm was proposed using a quadrature mixer in the receiving path. An analytical signal is formed at its output with real $I(t)$ and imaginary $Q(t)$ parts, similar to the Hilbert transform. It is then processed using a correlation procedure to calculate the current delay time $t_{d}$, after which the instantaneous Doppler frequency and velocity (2) are calculated. In this case, despite the heterogeneity of the signal in frequency and amplitude, you can accurately track the instantaneous value of the speed. The time $t_{d}$ corresponds to the maximum of the crosscorrelation function between $I(t)$ and $Q(t)$. By changing the parameters of the correlation signal processing, it is possible to measure almost instantaneous frequency in each of the two Doppler radar sensors. As a result, the conditionally instantaneous speed in each sensor can be determined by the formula:

$$
V_{\text {ins }}(t)=c f_{D} /\left[2 \cos (\alpha) \cos (\beta) f_{0}\right]=c /\left[8 t_{d} \cos (\alpha) \cos (\beta) f_{0}\right]
$$

\subsection{Direct displacement measurement with Doppler sensor}

Since the calculation process takes some time, and subsequent integration can also lead to the effect of error accumulation, it makes sense to switch to direct measurement of the displacement vector with some fixed discreteness error [4]. Due to this, it is possible to increase the accuracy and speed of measurements. The centers of the left and right tracks 
(projections) of the radiation patterns of the sensor antennas (see shaded areas in Fig. 1) will move along arcs with radii $R+r / 2$ and $R-r / 2$, where $\mathrm{r}$ is the distance between them. Then for some discrete same time period $T=\Delta t$, displacements along the arcs of these projections along the underlying surface $S_{1 i}$ and $S_{2 i}$ (see Fig.2) will be determined by the expressions:

$$
\left\{\begin{array}{l}
S_{1 i}=\pi\left(R_{i}+r / 2\right) \omega_{i} / 180^{\circ} \\
S_{2 i}=\pi\left(R_{i}-r / 2\right) \omega_{i} / 180^{\circ}
\end{array},\right.
$$

where $R_{i}$ and $\omega_{i}$ are the current radius and angle of rotation of the vehicle axis, which are determined from the solution of this system of equations (6):

$$
R_{i}=r\left(S_{1 i}+S_{2 i}\right) / 2\left(S_{1 i}-S_{2 i}\right), \quad \omega_{i}=\left(S_{1 i}-S_{2 i}\right) 180^{\circ} / \pi r .
$$

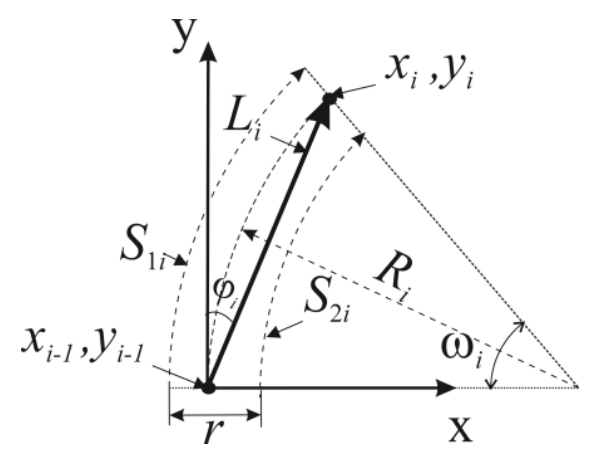

Fig. 2. Determination of the displacement vector $L_{i}$ when the vehicle is moving.

Since the chord length is $L_{i}=2 R_{i} \sin \left(\omega_{i} / 2\right)$, and $\varphi_{i}=\omega_{i} / 2$ (Fig. 4), taking into account (7), we obtain the expression for the array of displacement vectors $\left\{L_{i}, \varphi_{i}\right\}$ instead of velocity vectors $\left\{V_{i}, \varphi_{i}\right\}(3)$ :

$$
L_{i}=r\left(\frac{S_{1 i}+S_{2 i}}{S_{1 i}-S_{2 i}}\right) \sin \left(\frac{\left(S_{1 i}-S_{2 i}\right) 90^{\circ}}{\pi r}\right), \quad \varphi_{i}=\frac{\left(S_{1 i}-S_{2 i}\right) 90^{\circ}}{\pi r} .
$$

After that, immediately you can directly use the expression for permanent recurrent calculation of the array $\left\{\mathrm{x}_{i}, \mathrm{y}_{i}\right\}$ according to formulas (5). The problem of determining the displacements $S_{1 i}$ and $S_{2 i}$ actually consists in counting the number of periods of the Doppler signal for a constant quantization time interval $\Delta t_{i}$. The problem of determining the displacements actually consists in counting the number of half periods of the Doppler signal for a constant quantization time interval $\Delta t_{i}$. This can be done by counting the number of intersections of $I(t)$ and $Q(t)$ signals from the outputs of the quadrature mixer of each of the two Doppler sensors $-N_{1 i}$ and $N_{2 i}$ as shown in Fig.3. This is usually done using comparators.

Thus, $S_{1 i}$ and $S_{2 i}$ can be expressed as follows:

$$
S_{1 i}=\frac{\lambda_{0} N_{1 i}}{4 \cos (\alpha) \cos (\beta)}, \quad S_{2 i}=\frac{\lambda_{0} N_{2 i}}{4 \cos (\alpha) \cos (\beta)} .
$$

In this case, the displacement is measured with an error of up to half the wavelength of the Doppler signal, which corresponds to:

$$
\delta S= \pm \frac{\lambda_{0}}{4 \cos (\alpha) \cos (\beta)} .
$$


Figure 3 shows the signals from $I(t)$ and $Q(t)$ outputs of the quadrature mixer of one of the radar sensors and the pulse signal at the comparator output after these signals are fed to its inputs.
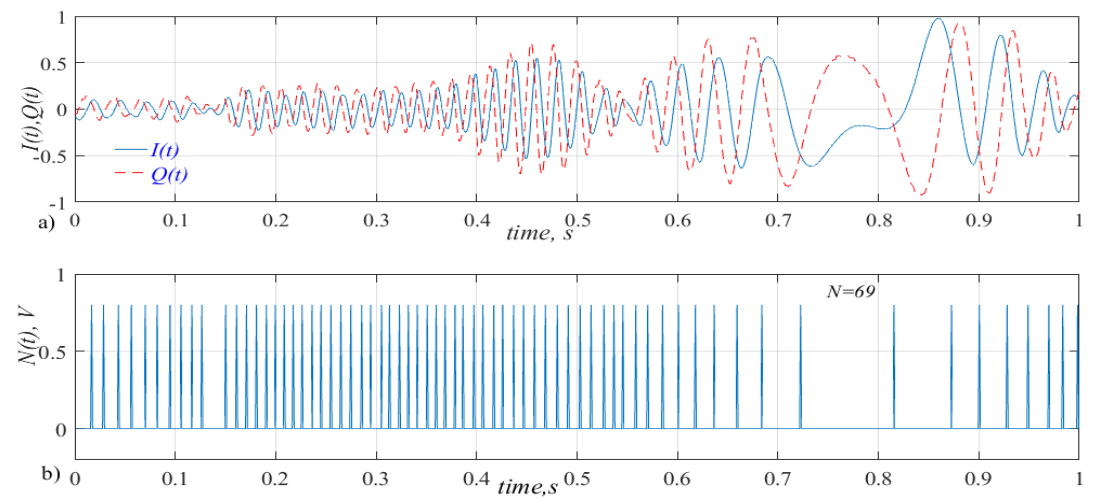

Fig. 3. Diagrams of the signals at the outputs of the quadrature mixer $I(t)$ and $Q(t)$ - a), and the pulses at the output of the comparator $N_{i}(\mathrm{t})$ - b).

In this example, in one second the number of half-periods $N$ is 69 . At a wavelength of $\lambda_{0}$ $=12.5 \mathrm{~mm}\left(f_{0}=24 \mathrm{GHz}\right), \alpha=\beta=45^{\circ}$, the path $S$ according to formulas (6) and (7) will be equal to $0.431 \mathrm{~m}$. It should be note that in this case, the accuracy of the path measurement does not change with sudden and frequent changes in the speed of movement of the object. In all other positioning methods this introduces a significant error.

\section{The advantages of direct measurement of displacement in ground positioning}

The radar navigation system, like any autonomous system, for example inertial, should be tied to the true coordinates on the ground. For this, a global satellite or other regional navigation system is used. With their help, the readings of the primary sensors are periodically adjusted. For an inertial system, these are data from accelerometers and gyroscopes. This procedure can be called the merging of autonomous and global navigation data, which is usually carried out using the Kalman filter [5].

\subsection{Using the Kalman filter to combine autonomous and global navigation data}

The Kalman filter model assumes that the state of the system during time $t$ evolved from its previous state at time $t-1$ in accordance with the equation

$$
\mathbf{x}_{t}=\mathbf{F}_{t} \mathbf{x}_{t-1}+\mathbf{B}_{t} \mathbf{u}_{t}+\mathbf{w}_{t},
$$

where $\mathbf{x}_{t}$ is a state vector containing system variables (position, speed, acceleration) at time $t, \mathbf{u}_{t}$ is a vector containing control actions. $\mathbf{F}_{t}$ is the state transition matrix that changes the system variables from the state of time $t-1$ to the state at time $t$. $\mathbf{B}_{t}$ is the control input matrix, $\mathbf{w}_{t}$ is a vector containing the process noise conditions for each parameter in the state vector. It is assumed that the process noise is taken from the zero mean multidimensional normal distribution with the covariance matrix $\mathbf{Q}_{t}$.

System measurements are also performed according to the model:

$$
\mathbf{z}_{t}=\mathbf{H}_{t} \mathbf{x}_{t}+\mathbf{v}_{t}
$$


where, $\mathbf{z}_{t}$ is the measurement vector, $\mathbf{H}_{t}$ is the matrix of the transformation of the state vector parameters into the measurement domain, $\mathbf{v}_{t}$ is the vector containing the noise measurement conditions for each observation in the state vector. The measurement noise is also considered to be zero mean Gaussian white noise with covariance $\mathbf{R}_{t}$.

The Kalman filter provides an algorithm for determining an estimate $\hat{\mathbf{x}}_{t}$ by combining system models and noisy measurements of certain parameters or linear parameter functions. Therefore, estimates of parameters of interest in the state vector are now provided by probability density functions $\mathbf{P}_{t}$, rather than discrete values. The Kalman filter algorithm includes two stages: forecasting and updating measurements. The standard equations for the prediction stage are

$$
\begin{aligned}
& \hat{\mathbf{x}}_{t \mid t-1}=\mathbf{F}_{t} \hat{\mathbf{x}}_{t-1 \mid t-1}+\mathbf{B}_{t} \mathbf{u}_{t} \\
& \mathbf{P}_{t \mid t-1}=\mathbf{F}_{t} \mathbf{P}_{t \mid t-1} \mathbf{F}_{t}^{T}+\mathbf{Q}_{t}
\end{aligned}
$$

The equations for the measurement change stage are given by the formulas:

$$
\begin{aligned}
& \hat{\mathbf{x}}_{t \mid t}=\hat{\mathbf{x}}_{t-1 \mid t-1}+\mathbf{K}_{t}\left(\mathbf{z}_{t}-\mathbf{H}_{t} \hat{\mathbf{x}}_{t \mid t-1}\right) \\
& \mathbf{P}_{t \mid t}=\mathbf{P}_{t \mid t-1}-\mathbf{K}_{t} \mathbf{H}_{t} \mathbf{P}_{t \mid t-1}
\end{aligned}
$$

Where $\mathbf{K}_{t}$ is the Kalman gain, which is determined from the formula:

$$
\mathbf{K}_{t}=\mathbf{P}_{t \mid t-1} \mathbf{H}_{t}^{T}\left(\mathbf{H}_{t} \mathbf{P}_{t \mid t-1} \mathbf{H}_{t}^{T}+\mathbf{R}_{t}\right)^{-1}
$$

\subsection{Advantages of autonomous navigation with direct motion measurement}

In the described merging algorithm, the state matrices of model $\left(\mathbf{x}_{t}, \mathbf{u}_{t}, \mathbf{F}_{t}, \mathbf{B}_{t}, \mathbf{Q}_{t}\right)$ are determined by the readings of the accelerometers and gyroscopes of the autonomous inertial system, and the measurement matrices $\left(\mathbf{z}_{t}, \mathbf{H}_{t}, \mathbf{R}_{t}\right)$ are determined by the data of the global positioning system. The state transition matrix $\mathbf{F}_{t}$ determines the predicted coordinates of the object after double (from accelerations) and single (from rotation speed) integration of INS readings. This leads to the rapid accumulation of computational errors over time. The $\mathbf{B}_{t}$ control matrix is strongly influenced by the mass of the object, friction against the ground, and air resistance. All this leads to a significant increase in the $\mathbf{Q}_{t}$ matrix, which makes the use of INS in land transport inefficient.

When using a radar positioning system instead of INS with direct measurement of the error, the bias of the accumulation of errors does not occur, and other errors in the $\mathbf{Q}_{t}$ matrix, which are characteristic of an inertial system during ground use, are also eliminated. Figure 4 shows a simulated route of a moving object, determined using the Kalman filter. In this case, a radar positioning system was used in conjunction with GNSS. Figure 5 shows the decrease in GNSS errors along the east and north axes when using the Kalman filter.

\section{Conclution}

The presented method of ranking radar ground vehicles can be used effectively instead of an autonomous inertial navigation system. Its advantage is the direct measurement of the displacement of the object by using microwave proximity sensors, which allows:

- to avoid the effect of accumulation of measurement errors in time characteristic of INS;

- to eliminate the errors associated with the mass of the object, the friction and resistance forces; 
— to simplify the calculations;

- continuously measure coordinates;

- to reduce the weight and cost of the sensors.

As a result, we can conclude that the use of microwave displacement sensors in the autonomous positioning of ground vehicles when working in combination with GNSS is promising.

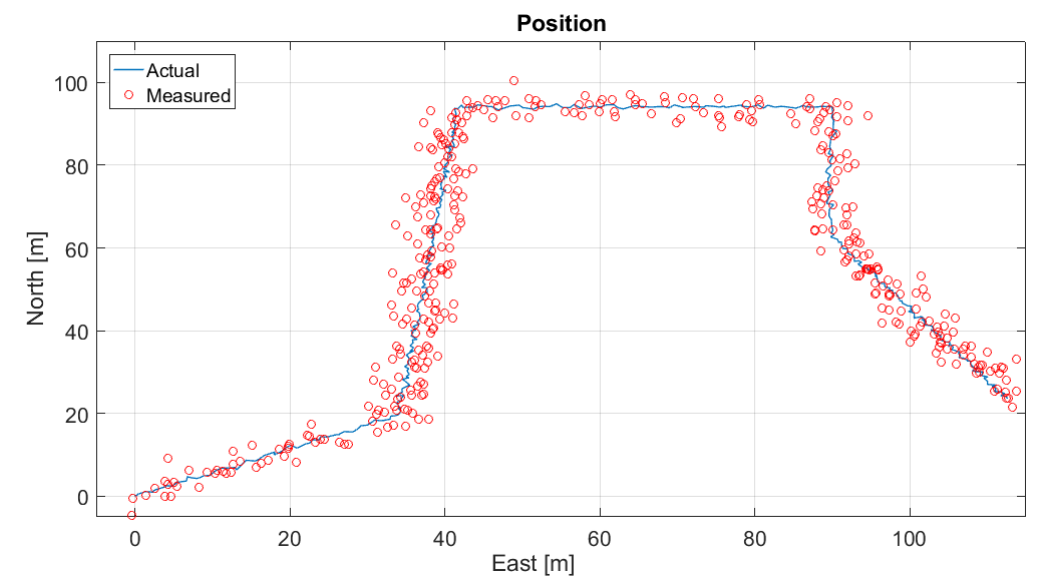

Fig. 4. Simulated route of a moving object, determined using the Kalman filter.
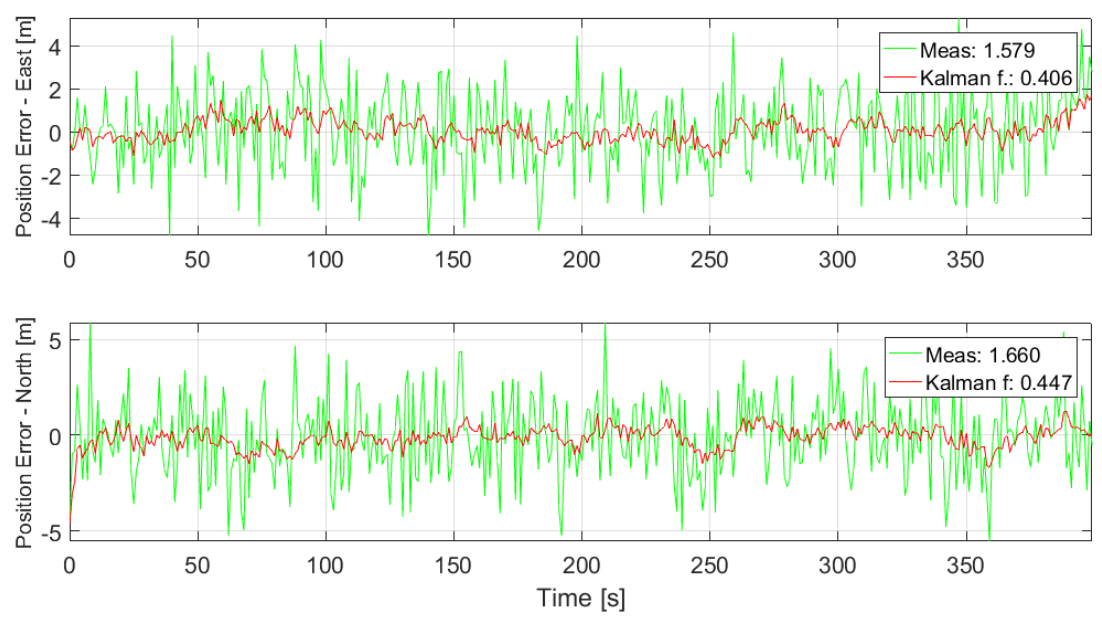

Fig. 5. GNSS errors along the east and north axes

\section{References}

1. D. Dardari, L. Marco, and E. Falletiis, Satellite and Terrestrial radio positioning thechniques. A signal processing perspective (Kidlington, Oxford, OX5, 1GB, UK, 2012)

2. D.V. Khablov Correlation Method for Signal Processing of Microwave Doppler Sensors, Measurement techniques, Vol. 60, No.5, pp. 505 -509 (2017)

3. D.V. Khablov Autonomous navigation system of ground transport based on Doppler sensors for measuring vector velocity, Measurement techniques, Vol. 61, No.4, pp. 384-389 (2018) 
4. D.V. Khablov Continuous ground positioning based on microwave displacement sensors // Izmeritelnaia technika, № 6, pp. 65-70 (2019)

5. R.E. Kalman A New Approach to Linear Filtering and Prediction Problems, Trans. of the ASME-Journal of Basic Eng., 82 (Series D): pp. 35-45 (1960) 